\title{
Semi-tacit Adaptation of Intelligent Environments
}

\author{
Tobias Heinroth $^{1}$, Achilles Kameas ${ }^{2}$, Hani Hagras ${ }^{3}$, Yacine Bellik ${ }^{4}$
}

1The Institute of Information Technology, Ulm University, Ulm, 89081 Germany (phone: 0049-731-50-26265; fax: 0049-731-50-26259; e-mail: tobias.heinroth@uni-ulm.de)

2The Hellenic Open University and DAISy research unit at the Computer Technology Institute, both in Patras, Hellas

3The Computational Intelligence Centre, Department of Computing and Electronic Systems, University of Essex, Wivenhoe Park, Colchester, CO43SQ, UK

4The National Center for Scientific Research (LIMSI-CNRS) BP 133, 91403, Orsay cedex, France and the Paris-South University

\begin{abstract}
This paper presents a semi-tacit adaptation system for implementing and configuring a new generation of intelligent environments referred to as adaptive ambient ecologies. These are highly distributed systems, which require new ways of communication and collaboration to support the realization of people's tasks. Semi-tacit adaptation is based on a mixed initiative approach in human-system dialogue management and is supported by three types of intelligent agents: Fuzzy Task Agent, Planning Agent and Interaction Agent. These agents use an ontology as a common repository of knowledge and information about the services and state of the ambient ecology.
\end{abstract}

\section{Introduction}

In the Ambient Intelligence (AmI) paradigm, intelligent computation will be invisibly embedded into our everyday environments through a pervasive transparent infrastructure (consisting of a multitude of sensors, actuators, processors and networks) which is capable of recognising, responding and adapting to individuals in a seamless and unobtrusive way [1].

We use the ambient ecology (AE) metaphor for Intelligent Environments realizing AmI concepts [2]. The vision of AmI aims at supporting people to carry out new tasks, as well as old tasks in new and better ways. People will realize their tasks using the resources offered by the services and devices of the ambient ecology. However, ambient ecologies are highly dynamic structures, the configuration of which may change, for example, because a new device may enter the ecology,

Please use the following format when citing this chapter:

Heinroth, T., Kameas, A., Hagras, H. and Bellik, Y., 2009, in IFIP International Federation for Information Processing, Volume 296; Artificial Intelligence Applications and Innovations III; Eds. Iliadis, L., Vlahavas, I., Bramer, M.; (Boston: Springer), pp. 423-429. 
or some other may cease functioning. While successful execution of tasks will depend on the quality of interactions among artefacts and among people and artefacts, it is important that task execution will still be possible, despite changes in the ambient ecology. Thus, the realization of mechanisms that achieve adaptation of system to changing context is necessary. In this paper, we present an adaptation mechanism that uses specialized intelligent agents (for task, plan and interaction adaptation) and a common repository of ecology knowledge and information in the form of an ontology, which is formed by matching the meta-data and selfdescriptions of the members of the ambient ecology. More specifically, we shall focus on a mixed initiative approach in human-system dialogue management that we call semi-tacit adaptation.

The remainder of this paper is structured as follows. Section 2 presents a scenario illustrating our concepts. Section 3 gives an overview of the basic modelling of an AE and presents the applications necessary to realize semi-tacit adaptation. The paper concludes in Section 4.

\section{Life in Intelligent Adaptive Homes}

In this section, we shall present a scenario based on the imaginary life of a user (Suki) who just moved to a home that is characterized by being intelligent and adaptive. The scenario will help to illustrate the concepts presented in the paper. To reference the different parts of the scenario in the other sections we use SP1...SPX as text marks.

Suki has been living in this new adaptive home for the past 10 months. Suki's living room has embedded in the walls and ceiling a number of sensors reading inside temperature and brightness; some more sensors of these types are embedded in the outside wall of the house. A touch screen mounted near the room entrance is used as the main control point. Suki uses an air-conditioning as the main heating / cooling device. The windows are equipped with automated blinds, which can be turned to dim or brighten the room. For the same purpose Suki can use the two lamps hanging from the ceiling. Finally, Suki has brought some hi-tech devices in the room: a digital flat screen TV set and a 9.1 sound system.

Suki's goal is to feel comfortable in his living room, no matter what the season or the outside weather conditions are. After careful thinking, he concluded that for him comfort involved the adjustment of temperature and brightness, the selection of his favourite TV channel and the adjustment of volume level, depending on the programme (SP1). Regarding the latter, the smart home system had observed Suki's choices over the past months and has drawn the conclusion that he tends to increase the volume when music or English speaking movies are shown, except when it's late at night; he keeps the volume low when movies have subtitles, or when guests are around (SP2). Nevertheless, the system does not have enough data to deduce Suki's favourite lighting and temperature conditions as the seasons change. Initially, the system will combine information in Suki's personal profile, 
the environmental conditions, the weather forecast and anything else that may matter, to tacitly adapt to the values that Suki might want. In case of a doubt, it will engage in dialogue with Suki about specific conditions. Of course, Suki can always set directly the values he desires by manipulating the devices that affect them; the system will monitor such activity and tacitly will adjust its rules.

For the past few days, as the weather has grown warmer, Suki has gone into a spring time mood; the system in his smart home has read the changing context and is trying to adapt, by decreasing the time that the heating system is on and by leaving the windows open for longer time intervals, during the sunny days (SP3). Suki still thinks that the living room is too warm and instructs the house to lower the temperature even further (SP4); the system, noticing that the day is sunny (it is early afternoon) and no rain or wind is foreseen for today, asks if Suki would prefer to open the windows, as well. Suki agrees, so the system opens the windows and lowers the thermostat only slightly. At the same time, it decreases slightly the volume of the TV set, as it may disturb the neighbours.

An hour later, Joan, Suki's friend arrives; she and Suki have arranged to go to a concert in the evening. Already the temperature has fallen and Suki asks the system to close the windows and lower the blinds; as a consequence, the system turns on the room lights, but Suki immediately switches it off. The system is puzzled and asks Suki if he wants some light or no light at all (SP5). Suki turns on a floor lamp that he bought only yesterday, but didn't have the chance to use until now (SP6). The system registers the new source of light and then asks Suki if this will be a new permanent brightness level, but Suki declines. After a while, Suki and Joan leave for the concert; the system shuts down all light and sound sources, but maintains the temperature until Suki's return.

\section{Modelling and Supporting Semi-tacit Adaptation}

An AE offers both physical properties and digital services and acts as a container for "activity spheres" (AS, see Fig. 1) [3]. An AS consists of passive entities (sensors, actuators, users, services, devices, etc.) and active entities namely Fuzzy Task Agent (FTA), Interaction Agent (IA) and Planning Agent (PA). It is intentionally created by an actor (human or agent) to support the realization of a specific goal. The sphere is deployed over an AE and uses its resources (artefacts, networks, services). The goal is described as a set of interrelated tasks; the sphere contains models of these tasks and their interaction. An AS is considered as a distributed yet integrated system that is formed on demand to support people's activities. It is adaptive in the sense that it can be instantiated within different environments and adaptively pursue its goals. An AS is realized as a composition of configurations between the artefacts and the provided services into the AE.

The configuration and the adaptation of a sphere could be realized in three ways, explicit, tacit and semi-tacit. In the former mode, people configure spheres by explicitly composing artefact affordances, based on the visualized descriptions 
of the artefact properties, capabilities and services [4]. In a highly dynamic system, such as an AS, explicit configuration is useful only for setting up the initial values or to model a default profile of the AS (see Fig. 2), because the huge number of interactions involved would impose a heavy cognitive load to the user, should he became aware of all of them. The tacit mode operates completely transparently to the user and is based on the system observing user's interactions with the sphere and actions within the sphere.

Agents in the intelligent environment can monitor user actions and record, store and process information about them [5], [6]. The sphere can learn user preferences and adapt to them, as it can adapt to the configuration of any new AE that the user enters. Tacit adaptation may achieve the opposite effect than the one it aims for, as people might feel that they have lost control over system's operation, which will appear incomprehensible and untrustworthy.

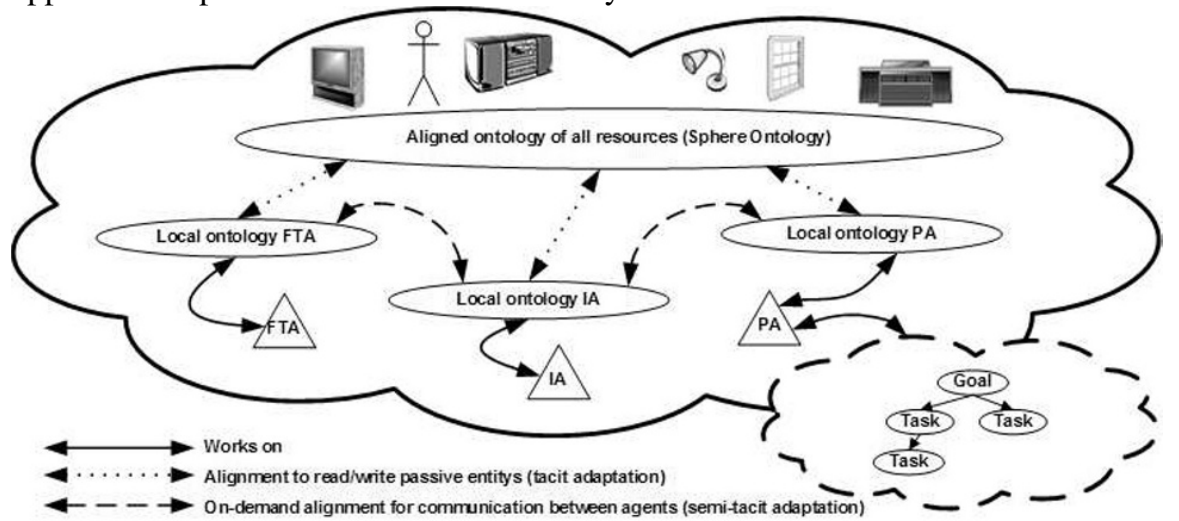

Fig. 1 Architectural overview of an activity sphere.

The semi-tacit mode realizes a third way of configuring and adapting the sphere by combining the explicit and the implicit way. The user interacts with the system, for example, using speech or screen-based dialogues. The user does not have to explicitly indicate resources to be used to program task models but the user can provide only basic information regarding his goals and objectives. The system, at the same time, attempts to tacitly resolve abstract tasks into concrete tasks and realize them, while monitoring user's actions with the resources involved. In case of rules that cannot be resolved, the system pro-actively engages into adaptive dialogue with the user.

The system uses the sphere ontology and three types of Agents: Planning Agent (PA), Fuzzy Task Agent (FTA) and Interaction Agent (IA). Each AS is composed by heterogeneous artefacts, each of which contains local descriptions of its services, which can be regarded as independent ontologies of varying complexity. to achieve efficient communication between the artefacts in the context of a task, we propose the application of ontology matching, to make the ontologies of the interacting artefacts semantically interoperable. Ontology matching is the process of finding relationships or correspondences between entities of different ontologies. 
This set of correspondences, is called an alignment [7]. So, by applying alignment algorithms to the local ontologies of the AE members, the user profile and the local agent ontologies, the sphere ontology is formed, which at each given moment, represents the collective knowledge required to realize a given task, as well as the state of the AS that supports this realization. Consequently, any adaptation mechanism needs to access the sphere ontology, not only for obtaining access to the correct $\mathrm{AE}$ resources, but also for obtaining the linguistic descriptions of the resources state.

To realize semi-tacit adaptation and thanks to the descriptive power of speech, we assume spoken dialogue interaction fits well to ask the user for further information to enhance planning or, if necessary, to resolve conflicts that may occur by negotiating with the user. One part of the IA is a speech dialogue manager (SDM) that receives problem descriptions from the PA and the FTA whenever semi-tacit adaptation is needed. Thus, the SDM tries to generate dialogues to react adequately.

The PA main task is to find out which tasks must be realized to support the user's activity and how they could be combined with the resources of the AE. During initialisation, the PA can use predefined rules and default domain models described in the task model. This initial information can either be pre-defined explicitly or in a semi-tacit way by utilising the IA to generate a (spoken) dialogue to retrieve general information from the user to personalize, for example, a default profile (see Fig. 2 and SP1). Then the system will form the sphere ontology by aligning the local ontologies of the members of the ecology, the agents and the user profile. But planning must also be done during the lifetime of the AS: The task model can be affected when conflicts arise (Suki behaves contrary to the system's consequences - SP5) or when a new device enters the ecology (SP6). In the first case it is usually not possible for the system to act completely tacitly so it tries to involve the user in a dialogue for conflict resolution. The latter case can normally be handled tacitly, by re-aligning the sphere ontology.

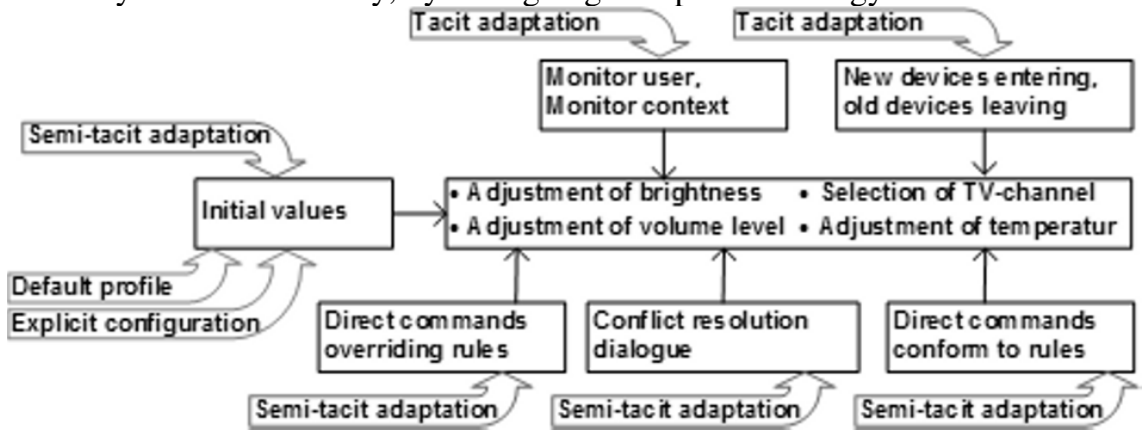

Fig. 2 Modes of configuration in ambient ecologies

The FTA will start to build an initial fuzzy logic based model of Suki's preferences and behaviour to realise the given tasks of maintaining the temperature, light levels and entertainment systems at the desired levels (SP2). To build the ini- 
tial model, the system will collect timestamped data (i.e. containing the related time and date) of the environment status (given temperature, light levels, user context (which room, activity, etc), the weather forecast, etc.) together with the user actions for such environment status (air-conditioning settings, blind settings, entertainment systems settings, etc). From the collected data, the FTA learns the needed fuzzy logic membership functions and rules needed to build the fuzzy logic model of Suki's preferences to realise the given task. The system then begins to adapt the generated fuzzy models over the short time interval to account for any environment of user behaviour changes. Over the long term, the fuzzy logic system will need to be adapted to Suki's change of preferences associated with seasonal variation (SP3). The FTA will then adapt its fuzzy logic systems rules and membership function to accommodate the faced uncertainties where the FTA will employ type-2 fuzzy logic systems [6]. The FTA acts completely in a tacit way similar to the PA as long as there are no conflicts or situations where the user is not satisfied with the adaptation (SP4).

\section{Conclusions}

In this paper, we presented a ubiquitous system architecture that achieves taskcentred adaptation using intelligent agents and ontologies. A task is modelled as part of a goal and is realized with the set of resources offered by an ambient ecology of smart artefacts and services. We call the combination of task descriptions and actual resources an activity sphere; then, the proposed architecture supports the execution of activity sphere applications. In the proposed architecture, adaptation is achieved with the help of an Interaction Agent, which engages in adaptive interaction with the user who is supported by the activity sphere. The system implements semi-tacit adaptation, in the sense that it achieves a balance between continuously interacting with the user and always acting on his behalf: by engaging in context-based proactive dialogue, the system attempts to maximize adaptation while minimizing the cognitive load imposed to the user. An example has been to illustrate the concepts and mechanisms that underlie the architecture.

Acknowledgement The research leading to these results has received funding from the European Community's Seventh Framework Programme (FP7/2007-2013) under grant agreement $n^{\circ}$ 216837 as part of the ATRACO Project (www.atraco.org).

\section{References}

1. K. Ducatel, M. Bogdanowicz, F. Scapolo, J. Leijten, and J.-C. Burgelman, "Scenarios for ambient intelligence in 2010”, IST Advisory Group Final Report, European Commission, February 2001. 
2. C. Goumopoulos and A. Kameas, "Ambient ecologies in smart homes", The Computer Journal, August 2008.

3. Zaharakis and A. Kameas, Engineering Emergent Ecologies of Interacting Artifacts, Handbook of Research on User Interface Design and Evaluation for Mobile Technology. 2008.

4. N. Drossos, C. Goumopoulos, and A. Kameas, "A conceptual model and the supporting middleware for composing ubiquitous computing applications", Journal of Ubiquitous Computing and Intelligence, vol. 1.

5. F. Doctor, H. Hagras, and V. Callaghan, "An intelligent fuzzy agent approach for realising ambient intelligence in intelligent inhabited environments", IEEE Transactions on System, Man \& Cybernetics, vol. 35.

6. H. Hagras, F. Doctor, A. Lopez, and V. Callaghan, "An incremental adaptive life long learning approach for type-2 fuzzy embedded agents in ambient intelligent environments", IEEE Transactions on Fuzzy Systems, vol. 15.

7. J. Euzenat and P. Schvaiko, Ontology Matching, 2007. 